\title{
Bioethik weltweit
}

\section{Jean Martin}

Dr. med., Mitglied der Redaktion

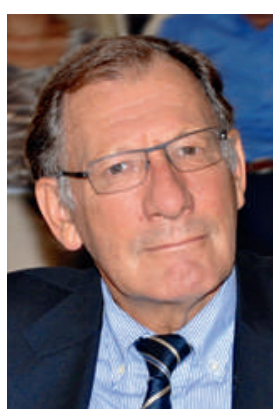

Ich beziehe das wöchentliche Online-Bulletin BioEdge (www.bioedge.org) und verfolge dort mit Interesse die Informationen zum weiten Thema der Bioethik. Der in Sydney wohnhafte Herausgeber Michael Cook, ein Wissenschaftsjournalist, unterhält eine zweite interessante Webseite namens Mercatornet (www.mercator net.com), deren Logo den Untertitel «Navigating modern complexities» trägt.

Die Artikel in BioEdge behandeln eine breite Themenpalette. Beispielsweise kommentiert Michael Cook dort die Enzyklika Laudato si von Papst Franziskus: "Laudato si ist gewissermassen eine umfassende Meditation über die Bioethik. Es bestand immer ein Spannungsfeld zwischen einer 'Bioethik der Freiheit', die den Akzent auf die Autonomie des Menschen und den Schutz seiner Integrität setzt, und einer 'globalen Bioethik', die das Schaffen des Menschen in seinem Umfeld, seiner Mitwelt miteinbezieht.» In diesem Sinne spricht Franziskus von integraler Ökologie.

BioEdge will zwar eine weltweite Perspektive entwickeln, spricht jedoch vor allem von Europa, Nordamerika und dem Südpazifik, wobei viele bioethische Arbeiten und Debatten aus diesen Regionen stammen. Euthanasie und Sterbehilfe werden inzwischen in vielen Ländern thematisiert. Im September 2015 wurde der Standpunkt von Richard Horton, Redaktor des Lancet, diskutiert, der einen wachsenden Konsens in der Akzeptanz der Sterbehilfe beobachtet. Und dies zu einem Zeitpunkt, wo das britische Parlament mit 3 zu 1 gegen eine Gesetzesänderung zur Legalisierung stimmt, der Gouverneur von Kalifornien hingegen ein Gesetz zur Legalisierung der Sterbehilfe in seinem Bundesstaat unterzeichnet.

Als dieser Artikel verfasst wurde, veröffentlichte BioEdge gerade eine Positionspapier des Internationalen Ausschusses für Bioethik der UNESCO: «Die Gentherapie markiert eine Wegscheide in der Medizin. $\mathrm{Ge}$ nome Editing (Selektion/Bearbeitung eines Genoms) ist ein vielversprechendes Unterfangen. Allerdings sind Eingriffe in das Genom nur zulässig zur Prävention, Diagnostik oder Therapeutik. Dabei dürfen diese Modifikationen nicht auf Nachkommen übertragbar sein.» Der IAB fordert eine umfassende Debatte zu diesem Potential der Wissenschaft und in dieser Phase ein Moratorium zum Genome Editing. In seiner jüngsten Arbeit meint der niederländisch-amerikanische Bio- ethiker Henk ten Have, für den ethische Verantwortung in der Realität der menschlichen Verletzlichkeit fusst, dass «angesichts der weltweiten Herausforderungen durch Armut, Ungleichheit und Umweltzerstörung der aktuelle bioethische Diskurs nicht mehr ausreicht. Er muss um eine breite Palette an Grundsätzen, die weit über die individuelle Perspektive hinausreichen, erweitert werden und unser Augenmerk auf die Verletzlichkeit als einer fundamentalen Komponente unseres Seins lenken [...]. Ethik erfüllt ihre Aufgabe nicht, wenn sie die soziale Dimension abstrahiert und die schädlichen Auswirkungen der Marktmechanismen auf das Gesellschaftsleben vernachlässigt.»

$\mathrm{Zu}$ den vielen angesprochenen Problemkreisen zählen: Die (fortgesetzte) Frage nach der Verweigerung aus Gewissensgründen in verschiedenen Bereichen, die medizinisch assistierte Fortpflanzung und ihre gelegentlich erstaunlichen Entwicklungen, Fragen zur Transplantation, zur sexuellen Nötigung von Chirurginnen in Australien ... Amüsantes Zitat vom 27. September: «Ziel der Transhumanisten ist die Verlängerung des Lebens auf unbestimmte Zeit. Nach Steve Fuller, einem führenden Soziologen und Professor an der Universität Warwick, ist es ein schwieriger Kampf, eine stupide Öffentlichkeit überzeugen zu wollen, die doch passiv die uns heute bevorstehende elende Zukunft akzeptiert» .. Ein Blick auf die Schweiz: Im August 2015 erschien ein Artikel über das Urteil des Bundesgerichts, in dem dieses einem homosexuellen Paar die Vaterschaft eines Kindes, das von einer Leihmutter im Ausland ausgetragen worden war, verwehrte. Im Mai wurde die Abstimmungsvorlage für den 14. Juni präsentiert (Verfassungsänderung zur Genehmigung der PID). Unser Land ist auch beim Thema Sterbehilfe mit dabei: 2014 verlangt ein Gesetz im Kanton Neuenburg von Alten- und Pflegeheimen, Sterbehilfemassnahmen zu akzeptieren. Zuvor stand im selben Kanton ein Arzt wegen Sterbehilfe vor Gericht und wurde freigesprochen.

Oft ist man enttäuscht über die Qualität von Artikeln zu Themen, mit denen wir uns auskennen ... Ich kann nicht alle Veröffentlichungen von BioEdge beurteilen, habe aber das Gefühl, dass die Texte und Kommentare weder ultraliberal noch zu konservativ sind. Generell scheinen sie mir fundiert und ausgewogen zu sein. Die Gewichtung und das sorgfältige Abwägen der Probleme sind wichtige Aufgaben der Ethik. 